\title{
ON INTERPRETING THE SUMS OF ASYMPTOTIC SERIES OF POSITIVE TERMS
}

\author{
J. E. DRUMMONDi
}

(Received 29 June 1981; revised 10 September 1981)

\begin{abstract}
Four different kinds of positive asymptotic series are identified by the limiting ratio of successive terms. When the limiting ratio is 1 the series is unsummable. When the ratio tends rapidly to a constant, whether greater or less than 1, the series is easily summed. When the ratio tends slowly to a constant not equal to 1 the series is compared with a binomial model which is then used to speed the convergence. When the ratio increases linearly, a limiting binomial and an exponential integral model are both used to speed convergence. The two resulting model sums are consistent and in this case are complex numbers. Truncation at the smallest term is found to be unreliable in the second case, invalid in the third case, and the exponential integral is used to produce a significantly improved truncation in the third case. A divergent series from quantum mechanics is also examined.
\end{abstract}

\section{Introduction}

Asymptotic series of positive terms occur in a number of perturbation expansions in quantum mechanics and other branches of physics. It may be possible to reformulate these calculations as has been described in the introduction to Drummond's [4] paper on the anharmonic oscillator, but it is the purpose of this paper to show that the existing series may also be transformed or interpreted to give the required answer.

There are in use a number of rational transformations of series, most of which have been reviewed by Smith and Ford [9], which effectively speed the convergence of both slowly convergent and divergent alternating series. However, when

\footnotetext{
'Department of Mathematics, Australian National University, P. O. Box 4, Canberra, A.C.T. 2600.

(c) Copyright Australian Mathematical Society 1982
} 
they are applied to series of positive terms they are found to have singularities within the operating range. An example of this is the Aitken $\delta_{2}$ transformation [2] also called by several other names including Shanks $e_{1}[7]$ and Padé $[0,1]$ (see, for example, $[3,6])$. This estimates the tail of a series $\sum_{r=n}^{\infty} u_{r}$ as $u_{n}^{2} /\left(u_{n}-u_{n+1}\right)$ but fails by going singular when $u_{n}=u_{n+1}$. Furthermore the Aitken $\delta_{2}$ transformation fails to sum $\Sigma 2^{n} / n$ to $-\ln (-1)$ or $\pm i \pi$. Other rational transformations have similar difficulties.

To overcome these difficulties two new methods are proposed for use in summing asymptotic series of positive terms. These are in addition to a sequence transformation with an imaginary parameter which gives comparable accuracy and has been recently published by Vanden Broeck and Schwartz [10].

The basic principle used in summing these series is to construct a model asymptotic series whose sum is known and whose terms behave in a manner similar to those of the unknown series. If the difference between the model and the series is convergent then the model sum of the series is defined as the sum for the model plus the difference between the series and the model. In doing this it has been found necessary to classify series according to the limiting behaviour of their term ratio $R_{n}$ (where $R_{n}=u_{n+1} / u_{n}$ and $u_{n}$ is a term of a series $S=\sum u_{n}$ ). An appropriate model is then assigned to each class of series.

This modelling technique is also used to find an improved truncation formula near the smallest term of a series.

\section{General considerations}

In the following we shall be mainly concerned with series of positive terms which either decrease in size from the start to a minimum then increase thereafter, or which increase throughout the series.

\subsection{Approximations to the remainder of an asymptotic series far from the smallest term}

Let $S_{n}=\sum_{r=0}^{n-1} u_{r}$, let $S$ be the asymptotic sum, let $u_{k}$ be the smallest term and suppose there exists an $\alpha$ such that $S_{k}+\alpha u_{k}=S$. Then, if $n>k$, the remainder after $n$ terms is $\left(S-S_{n}\right)$ where

$$
S-S_{n}=\alpha u_{k}-\sum_{r=k}^{n-1} u_{r} \doteqdot-u_{n-1}-u_{n-2}-u_{n-3}-\ldots
$$


If the terms increase rapidly in size while $n$ is greater than both $(k+\alpha)$ and $k$ then the remainder is approximately $-u_{n-1}$. Furthermore, if $u_{n-1}>u_{n-2}>$ $u_{n-3} \ldots$ and the series is approximately geometric, then a better approximation is

$$
-u_{n-1}-u_{n-2}-u_{n-3} \ldots \doteqdot-u_{n-1}^{2} /\left(u_{n-1}-u_{n-2}\right) \text { or }-u_{n-1} u_{n} /\left(u_{n}-u_{n-1}\right) \text {. }
$$

Similarly, if $n<k$ and $(k+\alpha)$, then

$$
S-S_{n}=\sum_{r=n}^{i-1} u_{r}+\alpha u_{k} \doteqdot u_{n}+u_{n+1}+u_{n+2}+\ldots
$$

This remainder is approximately $u_{n}$ or more accurately $u_{n-1} u_{n} /\left(u_{n-1}-u_{n}\right)$ or $u_{n}^{2} /\left(u_{n}-u_{n+1}\right)$ when $u_{n}$ decreases rapidly with $n$ and $R_{n}$ changes slowly along the series.

\subsection{Early truncation and the truncated sum}

THEOREM. If an asymptotic series of positive terms has a non-zero smallest term $u_{k}$ and an asymptotic sum $S$ which is greater than the first term of the series, then there exists an $n$ such that $S_{n} \leqslant S \leqslant S_{n+1}$.

PRoof. If every term $u_{r} \geqslant u_{k}>0$ then $S_{n}$, where $S_{n}=\sum_{r=0}^{n-1} u_{r}$, increases monotonically with $n$ without limit. Also $S_{1}$ is less than $S$. Hence there exists an $n$ such that $S_{n} \leqslant S \leqslant S_{n+1}$. Q.E.D.

If $u_{k}$ is the smallest term, this does not necessarily mean that $k=n$ or $(n+1)$ or that $|\alpha|<1$. Section 3.2 contains a counterexample where $|\alpha|=3.5$.

However, if $S$ exists and $\alpha$ is small but unknown, we may use equation (2) to carry out early truncation. Hence the error in $S_{n}$, where $n<k$, will be as given just below equation (2), even though the series may later diverge.

\subsection{Modelling and term splitting to estimate the model error}

Let $Z_{n}=u_{n}+u_{n+1}+u_{n+2}+\ldots$ be the tail of a series and let $T_{n}$ be the sum of the corresponding tail of a series which models the given series. Then, if the model coincides exactly with the series, $T_{r}=Z_{r}$ and $u_{r}+T_{r+1}=T_{r}$.

If the model is not exact we may still split each term $u_{r}$ of the series into two parts $T_{r}$ and $\left(u_{r}-T_{r}\right)$, then pair off the second part of each term with the first part of the next to form a new series,

$$
\begin{aligned}
Z_{n} & =\left(T_{n}+u_{n}-T_{n}\right)+\left(T_{n+1}+u_{n+1}-T_{n+1}\right)+\ldots \\
& =T_{n}+\left(u_{n}-T_{n}+T_{n+1}\right)+\left(u_{n+1}-T_{n+1}+T_{n+2}\right)+\ldots \\
& =T_{n}+\sum_{r=n}^{\infty} u_{r}^{*} \quad \text { where } u_{r}^{*}=u_{r}-T_{r}+T_{r+1} .
\end{aligned}
$$


If the original series is asymptotic and the early terms of the model are close to the early terms of the series, the new series will also be asymptotic and its smallest terms will enable us to estimate the accuracy of the model.

We now proceed to look at several asymptotic series, how they may be classified and suitable models for summing them.

\section{Modelling asymptotic series}

\subsection{The geometric series}

This is the basic series that all transformations must sum. The series

$$
S(x)=1+x+x^{2}+\ldots
$$

has an analytic continuation $-1 /(x-1)$ if $x>1$, which serves as a model for many divergent asymptotic series.

\subsection{The double exponential series}

This illustrates the minimum term, summability by the Shanks $e_{2}$ transformation [7] and the dangers of truncation.

Consider the series

$$
S=\sum_{r=0}^{\infty}\left(A a^{r}+B b^{r}\right) \quad \text { where } A, B>0, a>1 \text { and } 0<b<1 .
$$

This series is divergent and asymptotic but summable in the same sense as the geometric series. Also, if $A(a-1)<B(1-b)$ the series has a smallest term, which is a feature exhibited by a number of asymptotic series.

If we wish to sum this series by truncation then we first use the Shanks $e_{2}$ [7] transformation to express the remainder of the series, $Z_{n}=\sum_{r=n}^{\infty}\left(A a^{r}+B b^{r}\right)$, as a function of the first four terms of the remainder,

$$
Z_{n}=\frac{\left(u_{n+1} u_{n+2}-u_{n} u_{n+3}\right) u_{n}+\left(u_{n} u_{n+2}-u_{n+1}^{2}\right)\left(u_{n}+u_{n+1}\right)}{\left(u_{n+1} u_{n+3}-u_{n+2}^{2}\right)+\left(u_{n+1} u_{n+2}-u_{n} u_{n+3}\right)+\left(u_{n} u_{n+2}-u_{n+1}^{2}\right)} .
$$

This is also the $[1,2]$ Padé approximant (see, for example, $[3,6]$ ).

This formula may be simplified, if $a b$ is retained, to a three term formula for the remainder

$$
Z_{n}=\frac{u_{n}\left(a b u_{n-1}-u_{n}\right)}{a b u_{n-1}-(a b+1) u_{n}+u_{n+1}}
$$

Equations (4) and (5) may be verified by direct substitution. 
Equation (5) may now be used to find the best place to truncate an asymptotic series which behaves like a double exponential. Thus $S_{n}=S$ when $Z_{n}=0$ or $a b u_{n-1}=u_{n}$. This means that we should truncate a double exponential series just after $u_{n-1}$ where $a b u_{n-1}=u_{n}$. This is only at the smallest term when $a b=1$ or when the terms of the series are symmetric about the two equal smallest terms.

As a numerical example we sum the series

$$
S=\sum_{r=0}^{\infty}\left[(1.2)^{r}+8(0.6)^{r}\right]=9+6+4.32+\ldots
$$

The sum of the two G.Ps. is 15 . In equation (5), $a b=0.72$ and $u_{2}=a b u_{1}$. Hence $Z_{2}=0$ and the sum of the first two terms is equal to the geometric model sum, namely 15 . The equal smallest terms are the fifth and sixth, and the sum of five terms is 25.8864 which overestimates the model sum. The quantity $\alpha$ for equations (1) and (2) is -3.5 . The Aitken $\delta_{2}$ transformation is singular at the fifth term but, if started at the sixth term and repeated three times using eleven terms, the first terms of the successive transformations are approximately $25.8864-38.88+$ $24.83939+3.15843$, adding to 15.00422 but thereafter developing an initial instability. This instability can be avoided by starting the transformation later. Thus, if we use fifteen terms and transform four times starting with the eighth term, we obtain the sum 14.999977 ... correct to four decimal places. Some other rational formulae were tested and gave the same sum with comparable accuracies, while the Shanks $e_{2}$ and higher order Padé approximants sum the double exponential exactly.

\section{Summing a multiple exponential series}

Let $S=\sum_{r=0}^{\infty}\left(A a^{r}+B b^{r}+C c^{r}\right)$ where $a>b>c$. Such a series is identified by the fact that the ratio $R_{n}$ tends to a limit $a$ with exponentially small error as $n \rightarrow \infty$, it is divergent if $a>1$ and unsummable if $a, b$ or $c=1$.

These series are summable by any one of a number of rational transformations. For instance, if we split each term $u_{r}$ in the ratio $\left(-u_{r}: u_{r+1}\right)$ and recombine, then the new terms are $u_{r}^{*}$ where

$$
u_{r}^{*}=\frac{u_{r+1}\left(u_{r} u_{r+2}-u_{r+1}^{2}\right)}{\left(-u_{r}+u_{r+1}\right)\left(-u_{r+1}+u_{r+2}\right)},
$$

so

$$
u_{r}^{*} \simeq(a-b)^{2} B b^{r} /(a-1)^{2}
$$


This method of term splitting is again the Aitken $\delta_{2}$, Shanks $e_{1}$ or Padé $[0,1]$ transformation. It is singular if $u_{r}=u_{r+1}$ but if the transformation is started after the smallest term and used repeatedly on each previous transformation of the series, it rapidly sums any multiple exponential series in which $a, b$ and $c \neq 1$. The same applies to any of the other non-linear convergence speeding processes which approximate to a series by the ratio of two polynomials, provided we avoid the singularities of the process.

The Padé $[2,3]$ and higher order Padé approximations sum the triple exponential exactly. Even the constant weighted Euler transformation sums the series exactly in three steps if we know $a, b$ and $c$ and transform using the weights $a, b$ and $c$ successively in any order.

\section{Summing a polynomial series}

For a series whose general term is a polynomial of degree $k$ in $n, R_{n} \sim 1+k / n$ where $k$ is a non-negative integer. Such series may be slowly divergent but their sums are unbounded. To justify this, we note that any polynomial of degree $k$ can be expressed as a sum of factorials up to degree $k$, then we take as a model

$$
k !(1-x)^{-k-1}=\sum_{n=0}^{\infty}(n+1)(n+2) \cdots(n+k) x^{n} .
$$

If we set $x=1$ then the left hand side is unbounded.

$$
\therefore \sum_{n=0}^{\infty}(n+1)(n+2) \cdots(n+k) \text { is unbounded. }
$$

\section{Binomial and logarithmic series}

These series are identified by $R_{n} \sim a+b / n$ where $a>1$. The binomial and logarithmic functions have asymptotic series

$$
\begin{aligned}
(1-x)^{-p}= & 1+\int_{0}^{x} \frac{p d t}{(1-t)^{1+p}}=1+p x+\frac{p(p+1)}{2} x^{2} \ldots \\
& +\frac{(p+n-1) !}{(p-1) ! n !} x^{n}+\frac{(p+n) !}{(p-1) ! n !} \int_{0}^{x} \frac{(x-t)^{n} d t}{(1-t)^{n+p+1}}
\end{aligned}
$$


and

$$
-\ln (1-x)=\int_{0}^{x} \frac{d t}{1-t}=x+\frac{x^{2}}{2}+\cdots+\frac{x^{n}}{n}+\int_{0}^{x} \frac{(x-t)^{n} d t}{(1-t)^{n+1}} .
$$

The binomial series diverges if $x>1$ and has a smallest term if $p x<1$ while the $\log$ series diverges if $x>1$ and has a smallest term if $x<2$. Again the sum to the smallest term is unreliable because it is a real number and overestimates the sum of the series. On the other hand the binomial function can be recovered exactly, as follows, using any four adjacent terms of the series.

Let $Z_{0}=u_{0}+u_{1}+u_{2}+u_{3}+\ldots$ and

$$
A(1-x)^{-p}=\sum_{n=0}^{\infty} v_{n}
$$

where

$$
v_{n}=\frac{A p(p+1) \cdots(p+n-1)}{n !} x^{n}
$$

and

$$
u_{r}=v_{n+r}
$$

Then

$$
T_{0}=A(1-x)^{-p}-\sum_{r=0}^{n-1} v_{r}=\sum_{r=0}^{\infty} v_{n+r}=\sum_{r=0}^{\infty} u_{r} . \quad(6 \mathrm{a}, 6 \mathrm{~b}, 6 \mathrm{c})
$$

If we match the first four terms of the two series in equation (6c) and solve for $n, x, p$ and $A$, we obtain

$$
\begin{aligned}
& n=\left(-3 R_{2}+4 R_{1}-R_{0}\right) /\left(R_{2}-2 R_{1}+R_{0}\right), \\
& x=(n+2) R_{1}-(n+1) R_{0} \\
& p=-n+(n+1) R_{0} / x \\
& A=u_{0} n !(p-1) ! /\left((p+n-1) ! x^{n}\right)
\end{aligned}
$$

where $R_{n}=u_{n+1} / u_{n}$.

If $n$ is a positive integer we use equation (6a) to calculate $T_{0}$ and if $x>1$ we must use the analytic continuation from small $x$ round the branch point at $x=1$, so

$$
(1-x)^{-p}=(x-1)^{-p} e^{ \pm i p \pi} \text {. }
$$

If $p$ is a non-positive integer then $\Sigma v_{n}$ terminates, so we replace $(1-x)^{-p}$ by $(1-x)^{-p} \ln (1-x)$ in the above analysis.

If $n$ is not a positive integer we may still use the binomial model and interpolate or extrapolate to $n$ as follows. We first choose several integral values of $n$ near the calculated value of $n$ then for each value of $n$ calculate $x, p$ and $A$, 
which use the first three terms of the series. Hence we obtain several values of $T_{0}$ from equation (6a). Finally, we interpolate or extrapolate to the value of $T_{0}$ corresponding to the calculated value of $n$ or corresponding to zero difference in the values of $v_{n+3}$ and $u_{3}$.

If $T_{1}, T_{2}, T_{3}, \ldots$ are calculated similarly, the series for $Z_{0}$ is transformed as in equation (3) in Section 2.3. Apart from the first term, $T_{0}$, this will be an asymptotic series with smaller terms than the original series if the model is close to the original series.

\section{The exponential integral type series}

Asymptotic series for which $R_{n}$ increases linearly without limit are modelled by the exponential integral

$$
\begin{aligned}
e^{-x} \operatorname{Ei}(x) & =-\int_{-x}^{\infty} \frac{e^{-x-t}}{t} d t \\
& =\frac{1}{x}+\frac{1}{x^{2}}+\frac{2 !}{x^{3}}+\cdots+\frac{(n-1) !}{x^{n}}+(-1)^{n} \int_{-x}^{\infty} \frac{n ! e^{-x-t}}{t^{n+1}} d t,
\end{aligned}
$$

which is tabulated by Abramowitz and Stegun [1]. To find an approximate truncation formula we proceed as follows. If $x>1$ the smallest term of the series is $(n-1) ! / x^{n}$ where $n-1 \leqslant x \leqslant n$. If $n$ is close to $x$ we may choose the path of integration for the remainder integral to go from $-x$ to $-n$ then round a semicircle in the complex plane to $+n$ then to $\infty$. Hence the remainder is

$$
\begin{aligned}
-n ! \int_{x}^{n} \frac{e^{-x+z}}{z^{n+1}} d z & -\frac{n !}{n^{n}} \int_{0}^{\pi} e^{-x+n \cos \theta}[\sin n(\theta-\sin \theta)+i \cos n(\theta-\sin \theta)] d \theta \\
& +(-1)^{n} n ! e^{-2 x} \int_{n}^{\infty} \frac{e^{x-t}}{t^{n+1}} d t .
\end{aligned}
$$

If $n$ is close to $x$, if $u_{n}=n ! / x^{n+1}$ and if $e^{-2 x} \ll 1$, then the first integral is approximately $(x-n) u_{n}$, the second integral is approximately $u_{n}(-1 / 3 \pm i \sqrt{n \pi / 2})$ and the third integral is small.

If $u_{k-1}$ equals $(k-1) ! / x^{k}, u_{k}$ equals $k ! / x^{k+1}, u_{k+1}$ equals $(k+1) ! / x^{k+2}$ and $x$ is between $k$ and $(k+1)$ then $u_{k-1}, u_{k}$ and $u_{k+1}$ are the three smallest terms of the series. Furthermore, the function $\left(u_{k-1}-u_{k}\right) /\left(u_{k-1}-2 u_{k}+u_{k+1}\right)$ equals $x(x-k) /\left[(x-k)^{2}+k\right]$ and this varies almost linearly from 0 to 1 as $x$ varies from $k$ to $(k+1)$. The function $u_{k} /\left(u_{k-1}-2 u_{k}+u_{k+1}\right)$ equals $k x /\left[(x-k)^{2}\right.$ $+k]$. This equals $k$ when $x$ equals $k$ or $(k+1)$ and $u_{k-1} u_{k} /\left(u_{k-1} u_{k+1}-u_{k}^{2}\right)$ equals $x$. 
We may now evaluate the exponential integral by summing the series to the term $u_{k-1}$ just before the smallest term then substitute for $(x-k)$ and $k$ or $x$ $(\doteqdot k)$ from the three term expressions above. This gives us a three term expression for the remainder and hence a truncation formula with a correction term,

$$
\begin{aligned}
S \simeq & u_{0}+u_{1}+\cdots+u_{k-1}+\frac{\left(u_{k-1}-u_{k}\right) u_{k}-{ }_{3}^{1} u_{k}}{u_{k-1}-2 u_{k}+u_{k+1}} \\
& \pm i u_{k} \sqrt{\frac{\pi u_{k-1} u_{k}}{2\left(u_{k-1} u_{k+1}-u_{k}^{2}\right)}} .
\end{aligned}
$$

As a more precise alternative to equation (7) I wish to show in the following theorem that the limiting form of the truncated binomial as $n \rightarrow \infty$ and the truncated exponential integral series are equivalent. Hence the truncated binomial may be used as a model for the exponential integral.

THEOREM. If the rth terms of a truncated binomial series and an exponential integral series, truncated by starting at the nth and $k t h$ terms respectively, are

$$
v_{r}=\frac{A p(p+1) \cdots(p+n+r-1)}{(n+r) !} x^{n+r}, \quad u_{r}=\frac{(k+r-1) !}{z^{k+r}},
$$

and $u_{0}=v_{0}, u_{1}=v_{1}, u_{2}=v_{2}$ then for every $k$ and $r$

$$
\lim _{n \rightarrow \infty}\left(v_{r}-u_{r}\right)=0 \text {. }
$$

PROOF. If $u_{1} / u_{0}=v_{1} / v_{0}$ then $k(n+1)=(p+n) z x$.

If $u_{2} / u_{1}=v_{2} / v_{1}$ then $(k+1)(n+2)=(p+n+1) z x$.

$$
\begin{aligned}
\therefore z x=k+n+2 & \text { and } p+n=k(n+1) /(k+n+2) . \\
\therefore\left(u_{r+1} / u_{r}\right) \div\left(v_{r+1} / v_{r}\right) & =(k+r)(n+r+1) /((p+n+r) z x) \\
& =(k+r)(n+r+1) /(n k+n r+k r+2 r+k) .
\end{aligned}
$$

This tends to 1 as $n \rightarrow \infty$ for fixed $k$ and $r$. The constant $A$ may be chosen to make $u_{0}=v_{0}$ hence

$$
\lim _{n \rightarrow \infty}\left(v_{r}-u_{r}\right)=0 . \text { Q.E.D. }
$$

Note that as $n \rightarrow \infty, x \rightarrow \infty$ and $p \rightarrow-\infty$. 
As a numerical test of the rate of convergence of this result we use equations (6) to sum $\Sigma r$ !. If $n=2$, then $x=5, p=-7 / 5$ and $(1-5)^{7 / 5}=1-7+\frac{7.2}{1.2}\left(1+\frac{3}{3}+\right.$ $\left.\frac{38}{34}+\frac{3.813}{3.4 .5}+\cdots\right)$. Hence $1+1+2+\frac{26}{5}+\cdots=\frac{1}{7}\left(7-1-4^{7 / 5} e^{ \pm 7 \pi i / 5}\right) \simeq$ $.549697 \pm .946220 i$. Similarly, with $n=4,8$ and 16 the estimates are $.598896 \pm$ $1.020908 i, .638154 \pm 1.077026 i, .664351 \pm 1.112779 i$. If this sequence behaves like $a+b / n+c / n^{2}+d / n^{3}$ then

$$
a \simeq .6960 \pm 1.1548 i
$$

while

$$
e^{-1} \operatorname{Ei}(1) \simeq .697175 \pm 1.155727 i .
$$

Using more terms and larger values of $n$ will give closer agreement.

If we move one term along the same series and fit the second, third and fourth terms to a binomial with $n=3$, then $x=7, p=-13 / 7$ and

$$
\begin{aligned}
1+2+6+22+\cdots & =\frac{1}{13}\left[(1-7)^{13 / 7}-1+13-\frac{13.6}{1.2}\right] \\
& =-0.145380 \pm 0.930182 i .
\end{aligned}
$$

If $n=3,4,5,6$ and 7 the real parts of the sums are $-0.145380,-0.168122$, $-0.185185,-0.198439,-0.209020$. If these estimates behave like $a+b / n+c / n^{2}$ $+d / n^{3}+e / n^{4}$ then $a=-0.30075$, hence $(1+a)=0.699 \ldots$. Again, more and larger values of $n$ will improve the calculation.

In order to compare the truncation formula (7) and the binomial model for a series with a minimum we sum the series $\Sigma r ! / 5^{r+1}$.

Sum of five terms with zero $\alpha=0.273280$

Truncation with correction (Eq. 7) $=0.270720 \pm 0.02152 i$

Extrapolated binomial from

$$
\begin{aligned}
n=4,5,6,7 \text { and } 8 & =0.2707657 \pm 0.0211682 i \\
e^{-5} \operatorname{Ei}(5) & =0.2707662 \pm 0.0211678 i
\end{aligned}
$$

Simple truncation is least accurate and gives no imaginary component while the four term binomial is most accurate.

\section{Series for the Stark effect}

H. J. Silverstone [8] carried out some calculations on the Stark effect using a direct perturbation series. The terms of one of his series for the perturbed energy are

$$
(-5000-225-56-49-79-195-663-2992-17347) \times 10^{-4} \text { a.u. }
$$


where -0.5 atomic units (a.u.) is the normalised unperturbed energy, while the energy as given by Hehenberger et al. [5] is $-0.5275 \pm 0.00725 i$ a.u. The ratio of successive terms increases without apparent limit, so the series appears to be of the type described in Section 7. If we truncate before the smallest term the sum is -0.5281 while the truncation and interpolation formula (7) gives $-0.5275 \pm 0.0071 i$ and the binomial model using the four smallest terms gives the value $-0.5276 \pm$ $0.0073 i$.

Similar but more detailed calculations have been carried out by Drummond [4] for the anharmonic oscillator.

\section{Conclusion}

If one wishes to sum an asymptotic series of positive terms or say that the series represents a finite number, the first step should be to identify the type of series. The four different types of series described here must be treated differently and may be identified by the behaviour of the ratio $R_{n}\left(=u_{n+1} / u_{n}\right)$.

If $R_{n} \rightarrow 1$ from above or the terms are polynomials, no finite sum can be ascribed to the series.

If $R_{n}$ tends to a constant greater than 1 with exponentially small differences the tail may be summed by a number of non-linear convergence speeding processes before the instabilities of the smallest terms swamp the process.

If $R_{n}$ tends to a constant greater than 1 but converges slowly, fitting a binomial to three or four terms, and if necessary, using this for term splitting or interpolation may be successful.

The next case is of some use in quantum mechanics.

If $R_{n}$ increases linearly we may truncate just before the smallest term to obtain a rough estimate of the real part of $S$ or truncate with a correction to obtain a better estimate or match four terms of the series to a binomial series with large index.

In the first case truncation at the smallest term is invalid and in the second and third cases truncation at the smallest term can lead to serious errors.

\section{References}

[1] M. Abramowitz and I. A. Stegun (eds.), Handbook of mathemattcal functions (N.B.S. Appl. Math. Series 55, Washington, D. C., 1964).

[2] A. C. Aitken, "On Bernoulli's numerical solution of algebraic equations", Proc. Roy. Soc. Edinburgh 46 (1925-6), 289-305.

[3] G. A. Baker, Essentials of Pade approximants (Academic Press, New York, 1975). 
[4] J. E. Drummond, “The anharmonic oscillator; perturbation series for cubic and quartic energy distortion", J. Phys. Al4 (1981), 1651-1661.

[5] M. Hehenberger, H. V. McIntosh and E. Brändas, "Weyl's theory applied to the Stark effect in the hydrogen atom", Phys. Rev. A 10 (1974), 1494-1506.

[6] H. Padé, "Sur la représentation approchée d'une fonction par des fractiones rationelles", Ann. Ecole Norm. Superior Suppl. [3] 9 (1892), 1-93.

[7] D. Shanks, "Non-linear transformations of divergent and convergent sequences", J. Math. Phys. 34 (1955), 1-42.

[8] H. J. Silverstone, "Perturbation theory of the Stark effect in hydrogen to arbitrarily high order", Phys. Rev. A18 (1978), 1853-1864.

[9] D. A. Smith and W. F. Ford, "Acceleration of linear and logarithmic convergence", SIAM J. Numer. Anal. 16 (1979), 223-240.

[10] J. Vanden Broeck and L. W. Schwartz, "A one parameter family of sequence transformations", SIAM J. Math. Anal. 10 (1979), 658-666. 